\title{
Extracellular NME proteins: a player or a bystander?
}

\author{
Patrizia Romani ${ }^{1,4,5}$, Marilena Ignesti ${ }^{1,5}$, Giuseppe Gargiulo ${ }^{1}$, Tien $\mathrm{Hsu}^{2,3}$ and Valeria Cavaliere ${ }^{1}$
}

The Nm23/NME gene family has been under intensive study since Nm23H1/NME1 was identified as the first metastasis suppressor. Inverse correlation between the expression levels of NME1/2 and prognosis has indeed been demonstrated in different tumor cohorts. Interestingly, the presence of NME proteins in the extracellular environment in normal and tumoral conditions has also been noted. In many reported cases, however, these extracellular NME proteins exhibit anti-differentiation or oncogenic functions, contradicting their canonical anti-metastatic action. This emerging field thus warrants further investigation. In this review, we summarize the current understanding of extracellular NME proteins. A role in promoting stem cell pluripotency and inducing development of central nervous system as well as a neuroprotective function of extracellular NME have been suggested. Moreover, a tumor-promoting function of extracellular NME also emerged at least in some tumor cohorts. In this complex scenario, the secretory mechanism through which NME proteins exit cells is far from being understood. Recently, some evidence obtained in the Drosophila and cancer cell line models points to the involvement of Dynamin in controlling the balance between intra- and extracellular levels of NME. Further analyses on extracellular NME will lead to a better understanding of its physiological function and in turn will allow understanding of how its deregulation contributes to carcinogenesis.

Laboratory Investigation (2018) 98, 248-257; doi:10.1038/labinvest.2017.102; published online 16 October 2017

Cancer development depends on the interplay between oncogenes and tumor suppressor genes (TSGs). While tumor load can cause morbidity and weight loss, metastasis is the primary cause of patient mortality in the majority of cancers. ${ }^{1,2}$ Therefore the process of metastatic transformation and dissemination of cancer cells are under intensive studies. One particular area of interest involves the function of metastasis suppressor genes (MSGs).

Unlike TSGs, MSGs do not prevent primary tumor growth but inhibit cancer metastatization. The products of MSGs vary in their functions and subcellular locations, and have been detected in different intracellular compartments as well as in the extracellular environment. ${ }^{3}$

Nme1 was the first identified MSG, whose downregulation was found in metastatic derivative of a murine melanoma cell line. ${ }^{4}$ In human, the NME gene family consists of ten related members, some of them displaying the nucleoside diphosphate kinase activity (NDPK). ${ }^{5}$ NME1 and NME2 are the most closely related and are the ones most implicated in tumor progression. ${ }^{3}$ In many clinical cancer samples such as breast cancer, a correlation between a reduced NME level and metastasis has been found. ${ }^{6}$ However, in other tumors, upregulated NME levels have been correlated with poor prognosis, especially in neuroblastoma ${ }^{7}$ and some forms of leukemia and lymphoma. ${ }^{89}$ Therefore NME proteins are most likely multifunctional, and any therapeutics based on manipulating NME expression levels should consider the tissue context and potential side-effects. In this sense, the recently discovered extracellular activity of NME proteins necessitates closer examination of its pathophysiological relevance.

Although lacking a secretion signal peptide, high levels of extracellular NME proteins have been detected in tissue culture supernatants of a wide range of cancer cell lines and in body fluids of cancer patients. The association of extracellular NME proteins with tumor progression is of prognostic significance in a number of cancer types (see below). However, how these proteins can promote metastasis is still far from our understanding and is complicated by the intricate cellular and cancer stromal processes involved in metastasis.

In this review we first focus on involvement of the extracellular NME proteins as modulators of growth and

\footnotetext{
'Dipartimento di Farmacia e biotecnologie, Alma Mater Studiorum Università di Bologna, Bologna, Italia; ${ }^{2}$ Boston University School of Medicine, Department of Medicine, Boston, MA, USA and ${ }^{3}$ National Central University, Department of Biomedical Sciences and Technology, Jhongli, Taiwan

Correspondence: Professor T Hsu, PhD, National Central University, Department of Biomedical Sciences and Technology, No. 300 Jhongda Road, Jhongli 32001, Taiwan. E-mail: tienh@bu.edu or tienhsu@cc.ncu.edu.tw or Dr V Cavaliere, PhD, Alma Mater Studiorum Università di Bologna Farmacia e biotecnologie, Via Selmi, 3, Bologna, 40126, Italy. E-mail: valeria.cavaliere@unibo.it

${ }^{4}$ Current address: Dipartimento di Medicina molecolare, Università di Padova, Padova, Italia

${ }^{5}$ These authors contributed equally to this work.

Received 2 May 2017; revised 27 June 2017; accepted 20 July 2017
} 
differentiation of embryonic stem cells, and then we point out their putative significance in different tumor cells. In addition, we include our recent data obtained using the Drosophila model, on the regulation of extracellular levels of fly NME orthologs.

\section{EXTRACELLULAR NME1 AND NME7 IN STEM CELL PLURIPOTENCY REGULATION}

Stem cells are classified as totipotent, pluripotent, or multipotent. As the pluripotent stem cells possess the ability to become any cell type in the human body, they hold the greatest promise for therapeutic use. These cells could be used to replace damaged tissues in organs that have traditionally been thought not to have a significant potential for functional self-repair, such as brain tissue, heart muscle, kidney, and spinal cord. ${ }^{10-13}$ However, recent studies indicate that cultured human pluripotent stem cells are not truly pluripotent, rather they are in a slightly differentiated state called 'primed,' as opposed to the true pluripotent 'naive' state. ${ }^{14,15}$ In an attempt to create a growth system that enables maintenance of true pluripotency and self-renewal of naive stem cells, the Bamdad laboratory has demonstrated that NME acts as a growth factor interacting with its receptor $\mathrm{MUC1}^{\star}$ (a cleaved form of the full-length MUC1), promoting pluripotency and suppressing differentiation. ${ }^{16}$ The transmembrane protein MUC1 is a critical marker for identification of pluripotent stem cells as well as a key mediator of the growth and differentiation of stem cells. In particular, undifferentiated stem cells express a high level of the cleaved form of MUC1 $\left(\mathrm{MUC1}^{*}\right.$ ) that retains only 45 amino acids of the original extracellular domain and that has previously only been detected on cancer cells. ${ }^{17}$ NME1, which has been shown to be an activating ligand of $\mathrm{MUC1}^{\star}$ in cancer cells, colocalizes with $\mathrm{MUC1}^{*}$ on pluripotent stem cells. ${ }^{18}$ As soon as pluripotent cells initiate differentiation, the quiescent full-length form of MUC1 is detected. Thus, the switch from $\mathrm{MUCl}^{\star}$ to the full-length protein may be considered one of the first signals of the onset of differentiation.

Smagghe et al ${ }^{19}$ demonstrated through surface plasmon resonance that differential NME interaction with the extracellular domain of $\mathrm{MUC1}^{*}$ is strictly dependent on the NME multimerization state. The updated mechanistic model ${ }^{20}$ (Figure 1) suggests that early stem cells are able to secrete monomer NME7 that can bind to the extracellular domain of MUC1* and promotes growth and pluripotency. Hence, extracellular NME7 has an important role in maintenance of early stem cell in the naïve status. This is also supported by the evidence that master transcriptional regulators of pluripotency such as SOX2 and NANOG, are able to bind to the promoter of NME7. In addition, Wang et $a^{21}$ showed that Nme7 (and Nme6) expression levels are crucial for mouse embryonic stem cell renewal. Carter and colleagues also showed that in late stem cells, the pluripotency

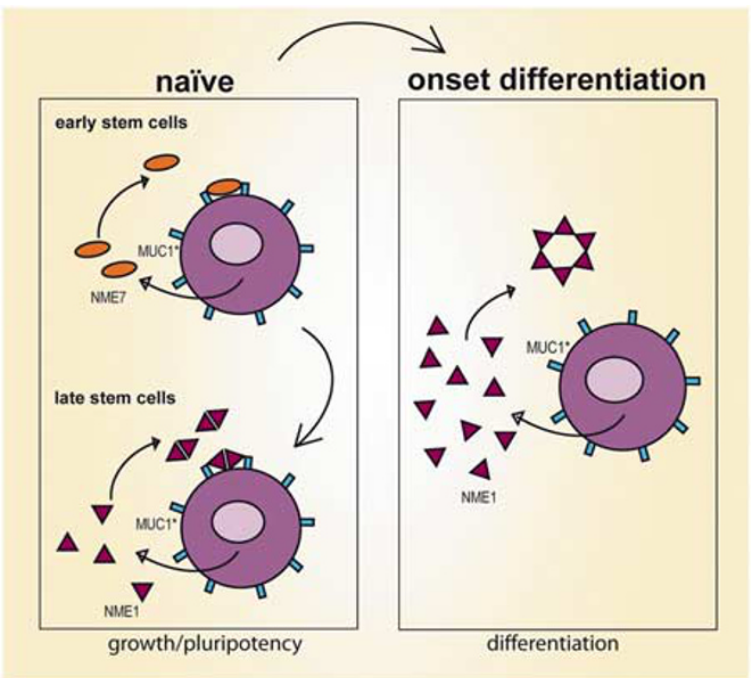

Figure 1 Mechanistic model illustrating how NME7 and NME1 control pluripotency and differentiation in stem cells. Modified from Carter et al. $^{20}$

transcription factor BRD4 (bromodomain and extraterminal domain family member) suppresses NME7 and upregulates NME1 that in turn replaces NME7 in promoting growth and pluripotency. Studies by the Bamdad laboratory compared the different binding affinities of NME1 multimers to the $\mathrm{MUC1}^{*}$ extracellular domain and demonstrated that only the NME1 dimeric form can bind to $\mathrm{MUC1}^{*} .{ }^{19}$ It is interesting that some NME1 mutants prefer the dimeric state. Of particular interest is the mutant $\mathrm{NME} 1^{\mathrm{S} 120 \mathrm{G}}$, which is the form mostly expressed in cancer cells. In this context, it is interesting to point out that cancer cells are characterized by their loss-of-ability to limit stem-like self-renewal. ${ }^{22,23}$ $\mathrm{NME1} 1^{\mathrm{S} 120 \mathrm{G}}$ in the dimeric form is sufficient to inhibit stem cell differentiation to allow long-term growth and maintenance of pluripotency and to increase expression of naïve markers.

Surprisingly, by manipulating the NME1 multimerization state, Smagghe et all ${ }^{19}$ also demonstrated that culturing undifferentiated stem cells in the presence of NME1 hexamers rapidly leads to differentiation. Interestingly, the affinitybinding assay demonstrated that NME1 hexameric form is not able to bind $\mathrm{MUC1}^{\star}$. The authors hypothesized that once the undifferentiated stem cells reach critical density, they secrete an additional amount of NME1 thus increasing its extracellular level in the microenvironment. At higher concentration extracellular NME1 forms hexamers that do not bind $\mathrm{MUCl}^{*}$ and differentiation occurs (Figure 1). It is not certain, however, whether hexameric NME1 can actively promote stem cell differentiation. Nonetheless, the implication is that extracellular NME proteins in different multimeric states exert opposite effects on stem cell differentiation and constitute a sort of ON/OFF switch. 


\section{EXTRACELLULAR NME1 IN CENTRAL NERVOUS SYSTEM PATHOPHYSIOLOGY}

Studies in mouse have pointed out a requirement of $\mathrm{Nmel}$ during the development of the nervous system. ${ }^{24,25}$ Most of the studies have focused on the role of intracellular Nmel; however, in 2010 Wright et $a l^{26}$ first reported that extracellular Nme1 is able to stimulate neurite outgrowth of explanted embryonic and adult dorsal root ganglia in vitro. The authors showed that extracellular Nmel acts as a positive chemotactic signal that induces neuronal growth cones to move toward substrates with higher Nmel concentration. It is worth noting that a more moderate but still significant outgrowth of neurites is observed even in the absence of the nerve growth factor. Moreover, the authors also observed enhanced neurite branching when dorsal root ganglia extend on Nmel-coated substrata, and growth cone turning at the boundary between Nmel-coated and uncoated substrata. Such directional movement of growth cones allows neurites to continue to grow in the microenvironment containing Nmel. Since this positive guidance effect of Nmel occurs in embryonic neurons, it could be hypothesized that the extracellular Nme1 also has a role in the development of the central nervous system (CNS). Indeed, chemotactic signals are broadly used during CNS development and, interestingly, evidence suggests that similar stimuli and subtended signaling pathways could also regulate vascular system development. ${ }^{27}$ The exact mechanism through which Nmel drives neurite outgrowth is not defined but the NDPK function seems not necessary, since extracellular kinase-dead mutant $\mathrm{Nmel}^{\mathrm{H} 118 \mathrm{~F}}$ is able to induce the same effect as the wild type protein.

Extracellular Nme1 has also been implicated in the shortterm response to damage in an in vitro traumatic brain injury model (TBI). Loov and colleagues co-cultured primary neurons, astrocytes and oligodendrocytes to simulate brain environment and analyzed, through mass-spectrometry, proteins present in the culture medium of injured and uninjured neurons. By comparing these proteomes, they found that Nmel was recovered as one out of 53 proteins exclusively present in the culture medium of injured neurons. ${ }^{28}$ This specific release after injury suggests a potential neuroprotective or regenerative function of extracellular Nme1. On the other hand, their data imply that neurons, astrocytes or oligodendrocytes do not secrete Nme1 in physiologically healthy conditions. However, whether the extracellular Nmel indeed plays an active role in neuron repair, or is a passive occurrence resulting from cell death, is not yet clear.

An established model for global brain insult is the postmortem cerebrospinal fluid (CSF) ${ }^{29}$ that has been successfully used to identify potential markers for neurodegeneration. Interestingly, NME1 is increased in CSF within $6 \mathrm{~h}$ postmortem with respect to control $\mathrm{CSF}^{29}$ suggesting the possibility that it could be a marker for neurodegenerative diseases. NME1 has also been identified as a highly sensitive and specific marker of stroke, whose plasma levels rise in the very early time frame of 3-6 h after the onset of symptoms. ${ }^{30}$ In this regard, the work from the Meloni laboratory ${ }^{31}$ in two different ischemia-related injury models (excitotoxicity mediated by L-glutamic acid and in vitro ischemia through oxygen and glucose deprivation) is noteworthy. They showed that exogenous Nmel treatment of primary rat cortical neuronal culture $24 \mathrm{~h}$ before and during the injuries enhances neuronal survival, thus exerting a neuroprotective activity. These authors speculated that this effect could be mediated through the binding of Nmel to a putative receptor thus triggering signaling pathway activation. The involvement of Mucl in this system is not likely because of the absence of expression of this glycoprotein in the brain. The authors also proposed an indirect mechanism of Nmel intervention for neuroprotection involving an enhancement of extracellular ATP or GTP levels leading to activation of purinergic receptor and triggering of neuroprotective pathways. It is possible that NME proteins might protect ADP and ATP from degradation by ectonucleotidases, but this mechanism would require one NME protein molecule for one nucleotide, which is highly inefficient. ${ }^{32}$ If as proposed, NME1 and NME2 can act as suppliers for extracellular ATP, this requires the classical transphosphorylase activity of NME proteins, which necessitates a constant source of NTPs and ADP to maintain ATP in effective concentrations. It has been shown that the amount of such nucleotides in the extracellar space is limited. ${ }^{33}$ This will also restrict the efficiency of extracellular NME proteins to generate nucleotide triphosphates even if more extracellular NME proteins are present. As such, these potential mechanisms require further validation.

Besides the hypothesized physiological roles in CNS development and regeneration, extracellular NME1 has also been linked to neuroendocrine tumor development such as neuroblastoma. Neuroblastoma is an embryonic malignancy arising from neural crest-derived progenitor cells, manifesting in pediatric patients. Interestingly, increased intracellular NME1 level has been linked to poor prognosis, contradicting its role as a metastasis suppressor. ${ }^{7}$ Moreover, serum level of NME1 also displays a direct correlation with poor outcome in neuroblastoma patients. ${ }^{34}$ Therefore intra- and extracellular NME1 may be pro-oncogenic in this setting, although it is not yet clear whether the extracellular NME1 has any pathophysiological function in this tumor type.

\section{EXTRACELLULAR NME IN ACUTE MYELOID LEUKEMIA}

Acute myeloid leukemia (AML) is an aggressive malignancy characterized by an uncontrolled clonal expansion of immature myeloid blast cells with an impaired capacity to differentiate. ${ }^{35}$ Proliferation of these cells in the bone marrow and blood affects normal hematopoiesis causing anemia, neutropenia and thrombocytopenia.

The central feature of AML is the failure of differentiation. Okabe-Kado and colleagues identified secreted Nme2 in murine M1 leukemic cell line that impaired myeloid 
differentiation. ${ }^{36,37}$ This activity was independent of its kinase function. $^{38}$ NME1 and NME2 were also found highly expressed in AML cells and the levels of NME1 gene expression was correlated with poor prognosis in AML..$^{39-41}$

The intracellular amount of NME1 was inversely correlated with hematopoietic maturation, ${ }^{42,43}$ suggesting an antidifferentiation function. The finding that NME1 is elevated in the serum of AML patients ${ }^{44}$ while no high concentration of this protein is present in normal healthy plasma, ${ }^{45}$ suggests a role of extracellular NME1 in AML. Therefore elevated expression of NME1 appears to be oncogenic in AML.

Data on the extracellular functions of NME proteins on AML cells came from using recombinant NME proteins (reviewed in Okabe-Kado et $a l^{46}$ and Lilly et $a l^{47}$ ). OkabeKado et $a l^{48}$ using recombinant NME proteins on cultured primary AML cells demonstrated that extracellular NME1 promoted the survival and growth of AML cells at a concentration equivalent to the level found in AML patients. This activity was independent of the NME1 NDPK function,

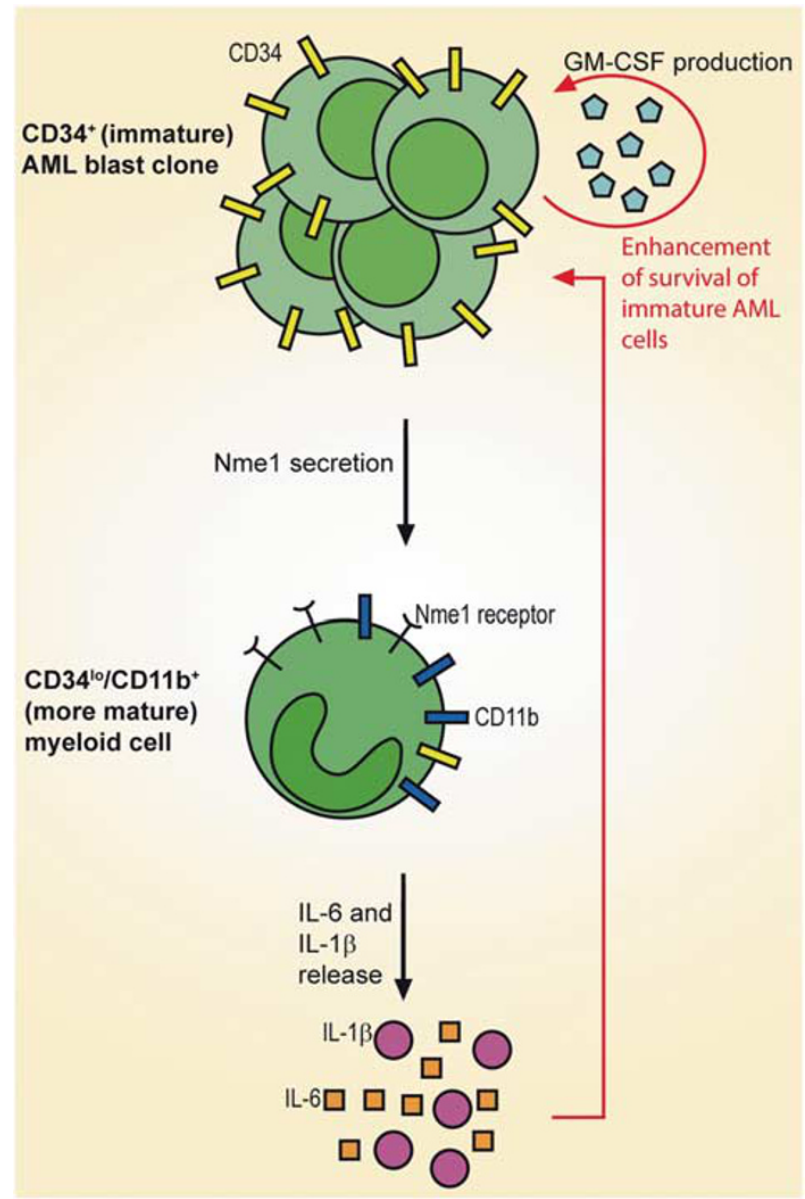

Figure 2 Model of the role of NME1 in the promotion of immature AML blast survival and growth. The interplay between subpopulations of AML cells at different maturity stages is mediated by the secretion of small molecules, including NME1. This generates a positive feedback loop that prompts malignant blasts to expand. Modified from Lilly et al. ${ }^{47}$ since the recombinant mutant $\mathrm{NME} 1^{\mathrm{H} 118 \mathrm{~F}}$ protein could promote survival and growth of AML cells. The pro-survival activity was also reported for the recombinant NME2 protein. ${ }^{48}$

Lilly et $a l^{49}$ by analyzing cells expressing the AML blast marker $\mathrm{CD}_{3} 4^{+}$and the myeloid differentiation marker $\mathrm{CD}_{11} \mathrm{~b}^{+}$demonstrated that NME1 does not bind the most immature blasts in AML mononuclear cell preparations, but it binds to more mature $\mathrm{CD} 34^{\mathrm{lo}} / \mathrm{CD} 34^{-}$(CD34low/negative) and $\mathrm{CD}_{11 \mathrm{~b}^{+}}$cells. Conditioned medium $(\mathrm{CM})$ by more mature cells that had been stimulated by NME1 enhances the survival of purified blast cells. These findings suggest that NME1 indirectly promotes survival and growth of AML blasts by acting on the more mature cells in the clone, and that a cross-talk between cell populations occurs within the tumor clone. However, the receptor responsible for the NME1 prosurvival effect on AML cells is still not known, and it has been shown that $\mathrm{MUC1}^{*}$ is not involved. ${ }^{49}$ How extracellular NME1 promotes survival and growth of AML cells is still unclear, but the response of AML cells to NME1 was associated with the production of a number of cytokines. An increased levels of granulocyte macrophage colony stimulating factor (GM-CSF), interleukin-1 $\beta$ (IL-1 $\beta$ ), and interleukin6 (IL-6) have been found in CM of AML cells treated with NME1. ${ }^{49,50}$ The cytokines GM-CSF and IL- $1 \beta$ are known to promote AML growth. ${ }^{51}$ Lilly et al ${ }^{47}$ used KGla CD34 ${ }^{+}$AML cell line, in which the high NME1 mRNA level is consistent with the one observed in primary $\mathrm{CD} 34^{+}$cells of $\mathrm{AML}$ samples. They demonstrated that NME1-containing CM and IL- $1 \beta$ could activate the nuclear factor- $\mathrm{KB}(\mathrm{NF}-\mathrm{\kappa B})$ that was shown to be constitutively active in AML stem cells. ${ }^{52}$ Altered activity of NFKB has been found in different types of cancer and is correlated with the cancer cell resistance to chemotherapies and radiation. ${ }^{53-56} 40 \%$ of AML patients have shown constitutively active $\mathrm{NF}-\mathrm{\kappa B}^{52}$ that allows leukemia cells to escape apoptosis and to proliferate. Based on their data the authors also suggest that $\mathrm{CD} 34^{+}$AML cells secrete GM-CSF after the release of IL-1 $\beta$ from NME1 stimulated mature $\mathrm{CD} 34^{\mathrm{lo}} / \mathrm{CD} 11 \mathrm{~b}^{+}$cells. Recently Lilly et al ${ }^{47}$ proposed a model on the function of NME1 in AML (Figure 2). The high expression of NME1 in the AML blast cells generates an elevated level of extracellular NME1, which binds to more mature myeloid cells promoting the secretion of a number of cytokines including IL- $1 \beta$ and IL- 6 . The role of IL-6 in AML is poorly understood; however, there is evidence indicating that high IL-6 levels trigger constitutive activation of the STAT3 pathway, which has been detected in $20-45 \%$ of AML samples. ${ }^{57,58}$ According to the model proposed by the authors, the secreted IL-1 $\beta$ and IL- 6 cytokines will promote the survival of blast cells, by triggering survival pathways such as STAT3 and NF- $\mathrm{kB}$, as well as enhancing the expression of other cytokines such as GM-CSF.

The overall findings on the extracellular NME1 activity on AML suggest that NME1 could be strongly involved in AML progression, not just a passive correlate of prognosis. 
Figure 3 Intra- and extracellular localization of Awd in Drosophila larval tissues. (a) Left panel, schematic drawing of the Drosophila wing disc (frontal view, up and z-stack, below). The red box and the red line indicate the position of the $x-y$ section and the $x-z$ section, respectively. Middle and right panels, confocal analysis of peripodal membrane and columnar cells stained for the DLG apical marker. In these panels, the dotted white lines indicate the position of confocal z-stack. By using the UAS-Gal4 system, ${ }^{96}$ we targeted the expression of UAS-Awd-GFP (green) transgene in peripodal cells under the control of the Ubx-Gal4 driver. Awd-GFP vesicles are detected in peripodal cells (arrow) and also in the lumen that separates these cells from the columnar epithelium (arrowhead). (b) Left panel, schematic drawing of the Drosophila fat body organ. Middle and right panels, immunofluorescence analysis of fat body tissue stained for Awd (red). By using the Flp-out/Gal4 technique, ${ }^{97}$ we induced clones of GFP positive adipocytes overexpressing $\mathrm{Shi}^{\mathrm{DN}}$, a dominant negative form of Shi. ${ }^{98}$ The white brackets enclose the clone. The white dotted lines in the $\mathrm{x}-\mathrm{y}$ section indicate the position of the $\mathrm{x}-$ $z$ projection magnification showed in lower panels. In Shi ${ }^{D N}$ clones, the intracellular Awd level is reduced. Scales are 20 and $5 \mu$ m for the $x-y$ and $x-z$ planar images, respectively. (c) Immunofluorescence analysis of fat body tissue stained for Awd (red). By using the MARCM technique ${ }^{99}$ we obtained cell clones (easily identifiable thanks to the presence of nuclear GFP) homozygous for $r a b 5^{2}$, an amorphic allele of rab5. The white dotted lines encircle a rab5 $5^{2}$ mutant adipocyte. There is no change of the Awd level in the rab5 $5^{2}$ mutant clone. Scale is $20 \mu \mathrm{m}$.

Moreover, of particular interest is that in addition to AML, high levels of NME1 were found in the serum of patients with malignant lymphomas, ${ }^{45,59-61}$ suggesting that the extracellular levels of this protein have a relevant role in clinical outcome in diverse hematological malignancies. ${ }^{46}$

\section{EXTRACELLULAR NME1 AND NME2 IN BREAST CANCER}

The presence of NME proteins in the extracellular environment of breast cancer cells has been investigated. Several in vitro studies have shown the presence of NME proteins in breast cancer cell CM. Anzinger et al ${ }^{62}$ have shown that the MDA-MB-231 metastatic human breast carcinoma cell line secretes NME2. The Buxton laboratory has also provided additional evidence of extracellular NME proteins in different tumor cell lines developed from women with metastatic breast cancer and from primary ductal carcinoma. These tumor cell lines are metastatic in the murine xenograft model. ${ }^{63}$ Western blot and NDPK activity analyses showed that NME1 and NME2 are present as soluble enzyme in growth medium of these tumor cell lines. These extracellular NME proteins promote endothelial cell migration and proliferation. The in vivo validation of NME1/2 presence in the extracellular environment has been obtained through western blot analyses of serum from women affected by different breast cancers. ${ }^{64}$

Recently, Izadpanah and Pucci-Minafra laboratories have performed proteomic profiling of extracellular vesicles secreted by the highly metastatic MDA-MB-231 breast cancer line and shown the presence of NME1 and NME2 in extracellular vesicles. ${ }^{65,66}$

Several studies have investigated the functional role of secreted NME and a possible role in purinergic signaling has emerged. Extracellular ATP has key roles as a neurotransmitter and as a signaling molecule. ${ }^{67}$ Once released, it is rapidly metabolized by various types of ectonucleotidases. ${ }^{68}$ Analyses of purine metabolism in the human bloodstream highlighted the existence of NDPK activity. ${ }^{32}$ Previous analyses also showed that the extracellular NDPK activity regulates extracellular nucleotide levels on the cellular surface in many cell types. ${ }^{33}$ Since the nucleotide amount in the extracellular space is limited, ${ }^{33}$ as discussed earlier the role of NME in modulation of ATP signaling by counteracting ectonucleotidases activity may depend on localized concentration of extracellular nucleotides.

Extracellular ATP/ADP and adenosine exert their biological roles by activating $\mathrm{P} 2$ and $\mathrm{P} 1$ receptor types, respectively. ${ }^{66}$ The P2Y family of ATP/ADP selective receptor is composed of heterotrimeric G protein-coupled receptors and includes eight different subtypes. The $\mathrm{P} 2 \mathrm{Y} 1$ and $\mathrm{P} 2 \mathrm{Y} 2$ receptors, in response to extracellular ATP, mediate proliferation in the vast majority of tumor types. Furthermore, activated P2Y1/2 receptors in turn transactivate the vascular endothelial growth factor type 2 receptor (VEGFR-2). ${ }^{69,70}$ This receptor transduces the vast majority of angiogenic and permeability effects of VEGF and thus has a crucial role in physiological as well as pathological conditions. ${ }^{71}$ Interestingly, many in vitro studies have shown that NME2 transactivates VEGFR-2. ${ }^{63,69,72,73}$ Yokdang et $a l^{63}$ have shown that treatments of human endothelial cell cultures with purified NME2 activates VEGFR-2 in a P2Y dependent fashion as pretreatment with MRS2179, a P2Y1-specific antagonist, blocked VEGFR-2 activation. Moreover, in vitro experiments have also shown that purified NME2 is able to promote P2Y1-dependent migration of endothelial cells.

Previous evidence obtained in the Buxton laboratory has already suggested a possible role of NME2 in supporting tumor formation by acting on extracellular ATP level. ${ }^{74}$ These in vitro experiments have shown that NME2 secreted from MDA-MB-435 human cancer cell line induces tubulogenesis of human cardiac endothelial cells. Significantly, they have also shown that MRS2179 treatment attenuates this in vitro pro-angiogenic effect. However it should be noted that the origin of MDA-MB-435 cell line has been determined to be melanoma; therefore this cell line should not be considered a model for breast cancer. ${ }^{75}$

Recently, Buxton laboratory has shown interesting results in a orthotopic xenograft model of breast cancer obtained through transplantation of MDA-MB-231 human breast cancer line in the mammary fat pat of SCID (severe combined immunodeficiency) mice. ${ }^{64}$ Monitoring of tumor growth and metastatization in this model has been possible thanks to the expression of the luciferase 2 gene in the MDA-MB-231(Luc+) transplanted cells. Besides to confirm the presence of NME1/2 proteins in the bloodstream, the analyses of this human breast 
a
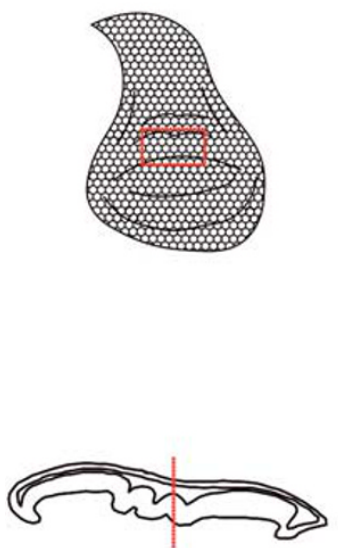

b

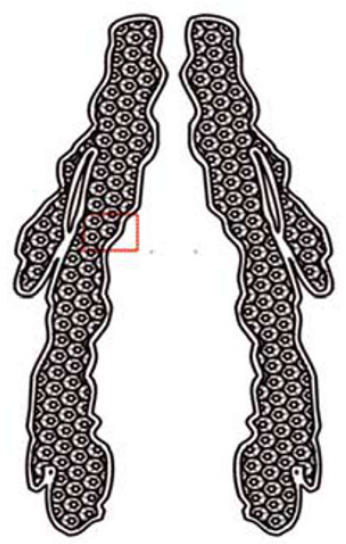

C
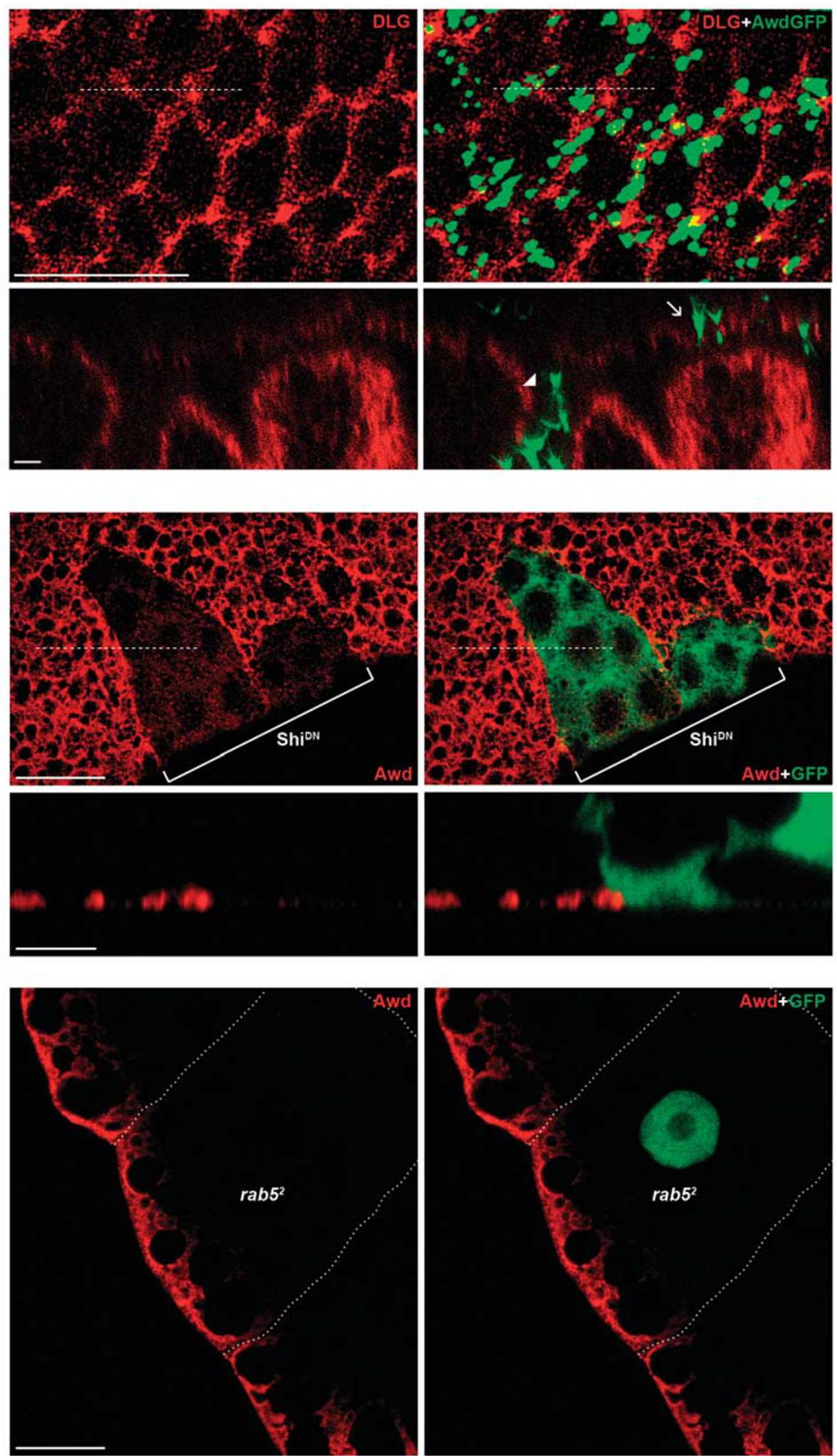
cancer xenograft have shown that increased tumor growth, monitored through in vivo imaging of bioluminescence, is correlated with enhanced level of circulating NME1/2. Furthermore, treatment of this orthotopic breast cancer model with the NDPK/VEGFR-2 inhibitor ellagic acid ${ }^{76,77}$ or with MRS2 $179^{78}$ reduced both primary tumor growth and metastasis to the lung.

Taken together, the extracellular NME proteins in CM of cell culture in vitro and in serum of patients or mouse cancer model seems to exert oncogenic effects.

\section{NME IN THE DROSOPHILA MODEL SYSTEM}

The abnormal wing discs (awd) is the unique Drosophila ortholog of the NME1 and NME2 proteins, sharing $78 \%$ of amino acid identity with human counterparts. ${ }^{79}$

Genetic studies unraveled an essential requirement of awd gene function at different stages during Drosophila melanogaster development. Indeed, awd is necessary for the development of the imaginal discs, ${ }^{80}$ the larval nervous system, ${ }^{24,80,81}$ the lymph gland ${ }^{80}$ (the fly hematopoietic organ) and the embryonic vascular system. ${ }^{82}$ Moreover, awd function during oogenesis has also been extensively explored. ${ }^{83-85}$ These studies support the notion that Awd fulfills a function as an endocytic mediator. In particular, awd genetically interacts with the well-characterized endocytic marker $R a b 5^{85}$ and with the internalization mediator shibire (shi), ${ }^{86}$ the fly homolog of the human Dynamin1 (DNM1). Beside its intracellular functions, interestingly, proteomic studies showed that Awd can be released into larval hemolymph, the unique Drosophila extracellular fluid, ${ }^{87}$ and into the extracellular environment by micro-vesicles in fly cell lines, ${ }^{88}$ suggesting potential extracellular roles of this NDPK. Recently, we showed that the balance between intracellular and extracellular Awd and NME1 is controlled by Shi and DNM1 in Drosophila and human cell lines. ${ }^{89-91}$ We studied Awd localization in the Drosophila wing disc primordium that is composed of a columnar proliferating epithelium in continuum with a peripodal membrane (Figure $3 \mathrm{a}$ ). In this tissue Awd is strictly localized in the apical region that faces the lumen, in vesicle-like aggregates that can be secreted by the wing disc epithelium to reach the hemolymph. By using transgenic lines expressing the GFP-tagged Awd, we observed that the wing disc is not the only tissue able to secrete Awd into the hemolymph. This finding suggests that the Awd balance between the outside and inside of the cell is highly dynamic. The extracellular Awd level is controlled by the Dynamin function since the extracellular protein level is enhanced in Drosophila larvae bearing a shi loss-of-function mutation $\left(s h i^{t s}\right)$. We also demonstrated that the relationship between extracellular NME1 and DNM1 exists in mammalian cells. By knocking down $d n m 1$ gene expression in normal colon (NCM) and colon carcinoma cancer (HT-29) cells, we observed a significant increase of extracellular NME1.
Larval hemolymph composition is mostly determined by the secretory and endocytic activities of adipocytes, polyploid cells composing the Drosophila larval fat body (scheme in Figure $3 \mathrm{~b}$ ). The fat body is a large endocrine organ that fulfills several functions resembling those performed by the mammalian liver. We showed that in adipocytes Awd protein is highly expressed (as also suggested by high-throughput awd expression data ${ }^{92}$ ) and it is mostly localized at the pericellular region. Interestingly, in adipocytes with impaired Shi activity, Awd intracellular level is greatly reduced (Figure 3b). This result is in accordance with the previously described enhancement of Awd amount in the hemolymph of $s h i^{\text {ts }}$ larvae and allows us to hypothesize that extracellular Awd is internalized by cells through a Shi-dependent mechanism. In order to further characterize Awd endocytic route, we analyzed the role of Rab5, the best-known early endosomal marker. We show that Awd trafficking into adipocytes is independent of Rab5 activity since its loss-of-function does not alter the intracellular level of Awd (Figure 3c).

The endocytic route NME1 follows during its internalization could have potential implications for cancer research. Indeed, Lim and colleagues ${ }^{93}$ presented an anti-metastatic therapy based on systemic delivery of a modified NME1 protein with improved cell-permeability. Their data support the notion that extracellular NME1 internalization by tumor cells inhibits metastasis-associated phenotypes in vitro, including cell migration, Matrigel invasion, ability to adhere to different substrates and ability to induce vascular endothelial cells to form angiogenic tube. Moreover, these authors also showed that treatment of tumor-bearing animals with the cell-permeable NME1 enhances their survival score and impedes the onset or stimulates the clearance of lung metastasis.

\section{CONCLUDING REMARKS AND PERSPECTIVE}

NME proteins have multiple cellular and molecular functions, some of which may contribute to their proposed antimetastatic activities. ${ }^{94}$ Moreover, these proteins may play different tissue-specific functions acting in various tumors and this could be the case also for the extracellular NME activity. As we highlighted in this review, growing evidence on the high extracellular NME levels in various tumor cohorts and in stem cell maintenance point out its possible function as pro-metastatic factor. However, it is not clear whether the elevated level of extracellular NME proteins indicates an active process used by the cancer cells to modulate the microenvironment, or it is a byproduct of tumor progression.

The most extensively studied extracellular NME protein functions are in the context of hematopoietic cells or pathophysiological conditions, including AML and cultured peripheral blood mononuclear cells. In these hematopoietic systems, extracellular NME proteins appear to inhibit differentiation, as is the case in stem cell maintenance. In other tumors, elevated NME proteins have been found in serum or body fluid in neuroblastoma and breast cancer 
patients. In breast cancer, extracellular NME proteins were shown to induce angiogenesis.

In light of their potentially metastasis-promoting effects, it is critical to know how wide-spread the presence of extracellular NME proteins is in other tumor patients. Currently the mechanism of NME protein secretion is unknown. It should be an important issue to address since an active secretory mechanism will support a pathophysiological role for the extracellular NME proteins. It would also be important to survey a range of different cancer cohorts to establish whether serum levels of NME proteins correlate with cancer prognosis using proteomic approaches.

Looking ahead, these are important to know before the implementation of therapeutics that elevate the expression of NME proteins based on their function as metastasis suppressor. However, besides the high relevance that the NME levels could serve as a prognostic and therapeutic targets of a specific tumor, the mode of action of the extracellular NME in normal and tumor cells is a challenging issue to be addressed. It has been shown that the NME NDPK activity is not required for the survival and growth of AML clones. Furthermore, in these cells NME1 may bind to an unknown receptor, different from the embryonic stem cells where it binds to $\mathrm{Mucl}^{\star}$ receptor. On the other hand, the oncogenic function of extracellular NME proteins in breast cancer requires their NDPK activity to modulate the purine levels.

The work on Drosophila model and in normal and colon carcinoma cells have suggested the involvement of Dynamin (therefore endocytosis) in internalizing extracellular NME proteins. Interestingly, these internalized extracellular NME proteins mediate the canonical metastasis suppressive function. ${ }^{90}$ From these data we may speculate that a mechanism balancing the intra- and extracellular NME levels in normal cells may be misregulated in cancer cells leading to a secretion of high levels of NME.

Taken together, the characteristics of extracellular NME proteins seem to mirror their intracellular counterparts, in terms of the complexity of physiological and molecular functions. Recently, it has been proposed that the myriad of intracellular NME functions could be explained by a model that NME proteins act as a scaffold, which exerts different functions in different subcellular locations based on the macromolecular partners (including proteins and lipids). ${ }^{95}$ It is tempting to speculate that the action of extracellular NME proteins follows the same rule.

In conclusion, the weight of the accumulating evidence seems to favor the notion that extracellular NME proteins do play important roles in pathophysiological conditions. Nonetheless, to clarify many of the unanswered questions, amenable in vivo models need to be established, in which a class of NME mutations should be created that specifically eliminates the extracellular NME. This will require the elucidation of the secretory mechanism. Future studies should focus on this point.

\section{ACKNOWLEDGMENTS}

The work was supported by a research grant from the Association for International Cancer Research (AICR-WWCR grant ref. 11-0738) to VC, a grant from University of Bologna (RFO 2013/2014) to GG and VC, a grant to TH from the National Institutes of Health, USA (\#R01CA109860), a grant to TH from Ministry of Science and Technology, Taiwan (MOST103-2320-B-008-002-MY3), and a grant to TH from National Health Research Institute, Taiwan (EX1050501BI). We also thank the Taiwan Bio-development Foundation (TBF) for the general support of the NCU research. Ml has been awarded a PhD fellowship from MIUR 'Fondo Giovani.' We also thank the Bloomington Stock Center for providing us with fly stocks and the Developmental Studies Hybridoma Bank at the University of lowa for monoclonal anti-DLG. We thank $L$ Shashidara for gift of the Ubx-Gal4 stock.

\section{DISCLOSURE/CONFLICT OF INTEREST}

The authors declare no conflict of interest.

1. Balmain A, Gray J, Ponder B. The genetics and genomics of cancer. Nat Genet 2003;33:238-244.

2. Haber DA, Settleman J. Cancer: drivers and passengers. Nature 2007;446:145-146.

3. Stafford L, Vaidya KS, Welch DR. Metastasis suppressors genes in cancer. Int J Biochem Cell Biol 2008;40:874-891.

4. Steeg PS, Bevilacqua G, Kopper L, et al. Evidence for a novel gene associated with low tumor metastatic potential. J Natl Cancer Inst 1988;80:200-204.

5. Desvignes $T$, Pontarotti $P$, Fauvel $C$, et al. Nme protein family evolutionary history, a vertebrate perspective. BMC Evol Biol 2009;9: 256.

6. Steeg PS, de la Rosa A, Flatow $\mathrm{U}$, et al. $\mathrm{Nm} 23$ and breast cancer metastasis. Breast Cancer Res Treat 1993;25:175-187.

7. van Noesel MM, Versteeg R. Pediatric neuroblastomas: genetic and epigenetic 'danse macabre'. Gene 2004;325:1-15.

8. Okabe-Kado J, Kasukabe T, Honma Y. Differentiation inhibitory factor $\mathrm{Nm} 23$ as a prognostic factor for acute myeloid leukemia. Leuk Lymphoma 1998:32:19-28.

9. Tschiedel S, Gentilini C, Lange T, et al. Identification of NM23-H2 as a tumour-associated antigen in chronic myeloid leukaemia. Leukemia 2008;22:1542-1550.

10. Mountford JC. Human embryonic stem cells: origins, characteristics and potential for regenerative therapy. Transfus Med 2008;18:1-12.

11. Odorico JS, Kaufman DS, Thomson JA. Multilineage differentiation from human embryonic stem cell lines. Stem Cells 2001;19:193-204.

12. Kroon E, Martinson LA, Kadoya K, et al. Pancreatic endoderm derived from human embryonic stem cells generates glucose-responsive insulin-secreting cells in vivo. Nat Biotechnol 2008;26:443-452.

13. Cho MS, Lee YE, Kim JY, et al. Highly efficient and large-scale generation of functional dopamine neurons from human embryonic stem cells. Proc Natl Acad Sci USA 2008;105:3392-3397.

14. Nichols J, Smith A. Naive and primed pluripotent states. Cell Stem Cell 2009:4:487-492.

15. Hanna J, Cheng AW, Saha K, et al. Human embryonic stem cells with biological and epigenetic characteristics similar to those of mouse ESCs. Proc Natl Acad Sci USA 2010;107:9222-9227.

16. Hikita ST, Kosik KS, Clegg DO, et al. MUC $1^{*}$ mediates the growth of human pluripotent stem cells. PLoS One 2008;3:e3312.

17. Mahanta S, Fessler SP, Park J, et al. A minimal fragment of MUC1 mediates growth of cancer cells. PLoS One 2008;3:e2054.

18. Nath S, Mukherjee P. MUC1: a multifaceted oncoprotein with a key role in cancer progression. Trends Mol Med 2014;20:332-342.

19. Smagghe BJ, Stewart AK, Carter MG, et al. MUC1* ligand, NM23-H1, is a novel growth factor that maintains human stem cells in a more naive state. PLoS One 2013;8:e58601.

20. Carter MG, Smagghe BJ, Stewart AK, et al. A primitive growth factor, $N M E 7 A B$, is sufficient to induce stable naive state human pluripotency; reprogramming in this novel growth factor confers superior differentiation. Stem Cells 2016;34:847-859.

21. Wang $\mathrm{C}-\mathrm{H}, \mathrm{Ma} \mathrm{N}$, Lin $\mathrm{Y}-\mathrm{T}$, et al. A shRNA functional screen reveals Nme6 and Nme7 are crucial for embryonic stem cell renewal. Stem Cells 2012;30:2199-2211. 
22. Kim YI, Park S, Jeoung DI, et al. Point mutations affecting the oligomeric structure of $\mathrm{Nm} 23-\mathrm{H} 1$ abrogates its inhibitory activity on colonization and invasion of prostate cancer cells. Biochem Biophys Res Commun 2003;307:281-289.

23. Chang $\mathrm{CL}$, Zhu $\mathrm{XX}$, Thoraval $\mathrm{DH}$, et al. Nm23-H1 mutation in neuroblastoma. Nature 1994;370:335-336.

24. Bilitou A, Watson J, Gartner A, et al. The NM23 family in development. Mol Cell Biochem 2009;329:17-33.

25. Carotenuto P, Marino N, Bello AM, et al. PRUNE and NM23-M1 expression in embryonic and adult mouse brain. J Bioenerg Biomembr 2006;38:233-246.

26. Wright KT, Seabright R, Logan A, et al. Extracellular Nm23H1 stimulates neurite outgrowth from dorsal root ganglia neurons in vitro independently of nerve growth factor supplementation or its nucleoside diphosphate kinase activity. Biochem Biophys Res Commun 2010;398:79-85.

27. Akita $\mathrm{T}$, Kumada $\mathrm{T}$, Yoshihara $\mathrm{S}$, et al. Ion channels, guidance molecules, intracellular signaling and transcription factors regulating nervous and vascular system development. J Physiol Sci 2016;66: 175-188.

28. Loov C, Shevchenko G, Geeyarpuram Nadadhur A, et al. Identification of injury specific proteins in a cell culture model of traumatic brain injury. PLoS One 2013;8:e55983.

29. Lescuyer P, Allard L, Zimmermann-Ivol CG, et al. Identification of postmortem cerebrospinal fluid proteins as potential biomarkers of ischemia and neurodegeneration. Proteomics 2004;4:2234-2241.

30. Allard L, Burkhard PR, Lescuyer P, et al. PARK7 and nucleoside diphosphate kinase $A$ as plasma markers for the early diagnosis of stroke. Clin Chem 2005;51:2043-2051.

31. Teoh J, Boulos S, Chieng J, et al. Erythropoietin increases neuronal NDPKA expression, and NDPKA up-regulation as well as exogenous application protects cortical neurons from in vitro ischemia-related insults. Cell Mol Neurobiol 2014;34:379-392.

32. Yegutkin GG, Samburski SS, Jalkanen S. Soluble purine-converting enzymes circulate in human blood and regulate extracellular ATP level via counteracting pyrophosphatase and phosphotransfer reactions. FASEB J 2003;17:1328-1330.

33. Lazarowski ER, Boucher RC, Harden TK. Constitutive release of ATP and evidence for major contribution of ecto-nucleotide pyrophosphatase and nucleoside diphosphokinase to extracellular nucleotide concentrations. J Biol Chem 2000;275:31061-31068.

34. Okabe-Kado J, Kasukabe T, Honma Y, et al. Clinical significance of serum NM23-H1 protein in neuroblastoma. Cancer Sci 2005;96: 653-660.

35. Saultz JN, Garzon R. Acute myeloid leukemia: a concise review. J Clin Med 2016;5

36. Okabe-Kado J. Factors inhibiting differentiation of myeloid leukemia cells. Crit Rev Oncog 1992;3:293-319.

37. Okabe-Kado J, Kasukabe T, Honma Y, et al. Identity of a differentiation inhibiting factor for mouse myeloid leukemia cells with NM23/ nucleoside diphosphate kinase. Biochem Biophys Res Commun 1992;182:987-994.

38. Okabe-Kado J, Kasukabe $T$, Hozumi M, et al. A new function of Nm23/ NDP kinase as a differentiation inhibitory factor, which does not require it's kinase activity. FEBS Lett 1995;363:311-315.

39. Wakimoto N, Yokoyama A, Okabe-Kado J, et al. Combined analysis of differentiation inhibitory factor $\mathrm{nm} 23-\mathrm{H} 1$ and $\mathrm{nm} 23-\mathrm{H} 2$ as prognostic factors in acute myeloid leukaemia. Br J Cancer 1998;77:2298-2303.

40. Yokoyama A, Okabe-Kado J, Sakashita A, et al. Differentiation inhibitory factor nm23 as a new prognostic factor in acute monocytic leukemia. Blood 1996;88:3555-3561.

41. Yokoyama A, Okabe-Kado J, Wakimoto $\mathrm{N}$, et al. Evaluation by multivariate analysis of the differentiation inhibitory factor nm23 as a prognostic factor in acute myelogenous leukemia and application to other hematologic malignancies. Blood 1998;91:1845-1851.

42. Willems R, Van Bockstaele DR, Lardon F, et al. Decrease in nucleoside diphosphate kinase (NDPK/nm23) expression during hematopoietic maturation. J Biol Chem 1998;273:13663-13668.

43. Yamashiro S, Urano T, Shiku $H$, et al. Alteration of $n m 23$ gene expression during the induced differentiation of human leukemia cell lines. Oncogene 1994;9:2461-2468.

44. Niitsu N, Okabe-Kado J, Nakayama M, et al. Plasma levels of the differentiation inhibitory factor $\mathrm{nm} 23-\mathrm{H} 1$ protein and their clinical implications in acute myelogenous leukemia. Blood 2000;96: 1080-1086.

45. Niitsu N, Okabe-Kado J, Kasukabe T, et al. Prognostic implications of the differentiation inhibitory factor $\mathrm{nm} 23-\mathrm{H} 1$ protein in the plasma of aggressive non-Hodgkin's lymphoma. Blood 1999;94:3541-3550.

46. Okabe-Kado J, Kasukabe T, Kaneko Y. Extracellular NM23 protein as a therapeutic target for hematologic malignancies. Adv Hematol 2012;2012:879368.

47. Lilly AJ, Khanim FL, Bunce CM. The case for extracellular Nm23-H1 as a driver of acute myeloid leukaemia (AML) progression. NaunynSchmiedeberg's Arch Pharmacol 2015;388:225-233.

48. Okabe-Kado J, Kasukabe T, Honma Y, et al. Extracellular NM23 protein promotes the growth and survival of primary cultured human acute myelogenous leukemia cells. Cancer Sci 2009;100:1885-1894.

49. Lilly AJ, Khanim FL, Hayden RE, et al. Nm23-h1 indirectly promotes the survival of acute myeloid leukemia blast cells by binding to more mature components of the leukemic clone. Cancer Res 2011;71: 1177-1186.

50. Okabe-Kado J, Kasukabe T, Honma $\mathrm{Y}$, et al. Extracellular NM23-H1 protein inhibits the survival of primary cultured normal human peripheral blood mononuclear cells and activates the cytokine production. Int J Hematol 2009;90:143-152.

51. Delwel $R$, van Buitenen C, Salem $M$, et al. Interleukin-1 stimulates proliferation of acute myeloblastic leukemia cells by induction of granulocyte-macrophage colony-stimulating factor release. Blood 1989;74:586-593.

52. Guzman ML, Neering SJ, Upchurch D, et al. Nuclear factor-kappaB is constitutively activated in primitive human acute myelogenous leukemia cells. Blood 2001;98:2301-2307.

53. Braunstein S, Formenti SC, Schneider RJ. Acquisition of stable inducible up-regulation of nuclear factor-kappaB by tumor necrosis factor exposure confers increased radiation resistance without increased transformation in breast cancer cells. Mol Cancer Res 2008;6:78-88.

54. Hoesel B, Schmid JA. The complexity of NF-kappaB signaling in inflammation and cancer. Mol Cancer 2013;12:86.

55. Napetschnig J, Wu H. Molecular basis of NF-kappaB signaling. Ann Rev Biophys 2013;42:443-468.

56. Perkins ND. The diverse and complex roles of NF-kappaB subunits in cancer. Nat Rev Cancer 2012;12:121-132.

57. Benekli M, Xia Z, Donohue KA, et al. Constitutive activity of signal transducer and activator of transcription 3 protein in acute myeloid leukemia blasts is associated with short disease-free survival. Blood 2002;99:252-257.

58. Schuringa JJ, Wierenga AT, Kruijer W, et al. Constitutive Stat3, Tyr705, and Ser727 phosphorylation in acute myeloid leukemia cells caused by the autocrine secretion of interleukin-6. Blood 2000;95: 3765-3770.

59. Niitsu N, Honma $\mathrm{Y}$, lijima K, et al. Clinical significance of nm23-H1 proteins expressed on cell surface in non-Hodgkin's lymphoma. Leukemia 2003;17:196-202.

60. Niitsu N, Nakamine H, Okamoto M, et al. A clinicopathological study of $\mathrm{nm} 23-\mathrm{H} 1$ expression in classical Hodgkin's lymphoma. Ann Oncol 2008;19:1941-1946.

61. Niitsu N, Okamoto M, Okabe-Kado J, et al. Serum nm23-H1 protein as a prognostic factor for indolent non-Hodgkin's lymphoma. Leukemia 2001;15:832-839.

62. Anzinger J, Malmquist NA, Gould J, et al. Secretion of a nucleoside diphosphate kinase $(\mathrm{Nm} 23-\mathrm{H} 2)$ by cells from human breast, colon, pancreas and lung tumors. Proc West Pharmacol Soc 2001;44:61-63.

63. Yokdang $\mathrm{N}$, Tellez JD, Tian $\mathrm{H}$, et al. A role for nucleotides in support of breast cancer angiogenesis: heterologous receptor signalling. $\mathrm{Br} \mathrm{J}$ Cancer 2011;104:1628-1640.

64. Yokdang N, Nordmeier S, Speirs K, et al. Blockade of extracellular NM23 or its endothelial target slows breast cancer growth and metastasis. Integr Cancer Sci Ther 2015;2:192-200.

65. Kruger S, Abd Elmageed ZY, Hawke DH, et al. Molecular characterization of exosome-like vesicles from breast cancer cells. BMC Cancer 2014;14:44.

66. Palazzolo G, Albanese NN, DIC G, et al. Proteomic analysis of exosomelike vesicles derived from breast cancer cells. Anticancer Res 2012;32: 847-860.

67. Burnstock G. Purinergic signalling: Its unpopular beginning, its acceptance and its exciting future. Bioessays 2012;34:218-225. 
68. Yegutkin GG. Enzymes involved in metabolism of extracellular nucleotides and nucleosides: functional implications and measurement of activities. Crit Rev Biochem Mol Biol 2014;49: 473-497.

69. Rumjahn SM, Yokdang N, Baldwin KA, et al. Purinergic regulation of vascular endothelial growth factor signaling in angiogenesis. $\mathrm{Br} J$ Cancer 2009;100:1465-1470.

70. Seye $\mathrm{Cl}, \mathrm{Yu} \mathrm{N}$, Gonzalez FA, et al. The P2Y2 nucleotide receptor mediates vascular cell adhesion molecule-1 expression through interaction with VEGF receptor-2 (KDR/Flk-1). J Biol Chem 2004;279: 35679-35686

71. Shibuya M. Vascular endothelial growth factor and its receptor system: physiological functions in angiogenesis and pathological roles in various diseases. J Biochem 2013;153:13-19.

72. Feng $\mathrm{Y}$, Gross S, Wolf NM, et al. Nucleoside diphosphate kinase B regulates angiogenesis through modulation of vascular endothelial growth factor receptor type 2 and endothelial adherens junction proteins. Arterioscler Thromb Vasc Biol 2014;34:2292-2300.

73. Wieland $\mathrm{T}$. Interaction of nucleoside diphosphate kinase B with heterotrimeric $\mathrm{G}$ protein betagamma dimers: consequences on $\mathrm{G}$ protein activation and stability. Naunyn-Schmiedeberg's Arch Pharmacol 2007;374:373-383.

74. Rumjahn SM, Javed MA, Wong $\mathrm{N}$, et al. Purinergic regulation of angiogenesis by human breast carcinoma-secreted nucleoside diphosphate kinase. Br J Cancer 2007;97:1372-1380.

75. Holliday DL, Speirs V. Choosing the right cell line for breast cancer research. Breast Cancer Res 2011;13:215.

76. Buxton IL. Inhibition of $\mathrm{Nm} 23 \mathrm{H} 2$ gene product (NDPK-B) by angiostatin, polyphenols and nucleoside analogs. Proc West Pharmacol Soc 2008;51:30-34.

77. Wang N, Wang Z-Y, Mo S-L, et al. Ellagic acid, a phenolic compound, exerts anti-angiogenesis effects via VEGFR-2 signaling pathway in breast cancer. Breast Cancer Res Treat 2012;134:943-955.

78. Shen J, DiCorleto PE. ADP stimulates human endothelial cell migration via P2Y1 nucleotide receptor-mediated mitogen-activated protein kinase pathways. Circ Res 2008;102:448-456.

79. Rosengard AM, Krutzsch HC, Shearn A, et al. Reduced Nm23/Awd protein in tumour metastasis and aberrant Drosophila development. Nature 1989;342:177-180.

80. Dearolf CR, Hersperger E, Shearn A. Developmental consequences of $a w d b^{3}$, a cell-autonomous lethal mutation of Drosophila induced by hybrid dysgenesis. Dev Biol 1988;129:159-168.

81. Timmons L, Hersperger E, Woodhouse $\mathrm{E}$, et al. The expression of the Drosophila awd gene during normal development and in neoplastic brain tumors caused by lgl mutations. Dev Biol 1993;158:364-379.

82. Dammai V, Adryan B, Lavenburg KR, et al. Drosophila awd, the homolog of human $n m 23$, regulates FGF receptor levels and functions synergistically with shi/dynamin during tracheal development. Genes Dev 2003;17:2812-2824.

83. Ignesti $M$, Barraco $M$, Nallamothu $G$, et al. Notch signaling during development requires the function of awd, the Drosophila homolog of human metastasis suppressor gene Nm23. BMC Biol 2014;12:12.
84. Nallamothu G, Woolworth JA, Dammai V, et al. awd, the homolog of metastasis suppressor gene $N m 23$, regulates Drosophila epithelial cell invasion. Mol Cell Biol 2008;28:1964-1973.

85. Woolworth JA, Nallamothu G, Hsu T. The Drosophila metastasis suppressor gene Nm23 homolog, awd, regulates epithelial integrity during oogenesis. Mol Cell Biol 2009;29:4679-4690.

86. Krishnan KS, Rikhy R, Rao S, et al. Nucleoside diphosphate kinase, a source of GTP, is required for dynamin-dependent synaptic vesicle recycling. Neuron 2001;30:197-210.

87. de Morais Guedes S, Vitorino R, Domingues $\mathrm{R}$, et al. Proteomics of immune-challenged Drosophila melanogaster larvae hemolymph. Biochem Biophys Res Commun 2005;328:106-115.

88. Koppen T, Weckmann A, Muller S, et al. Proteomics analyses of microvesicles released by Drosophila Kc167 and S2 cells. Proteomics 2011;11:4397-4410.

89. Hsu T, Romani P, Papi A, et al. Extracellular NME proteins-a long and still controversial story. In: Edited by Ćetković $\mathrm{H}$, Bosnar $\mathrm{MH}$. 10th International Congress of the NDP Kinase/Nm23/Awd Family (NDPK2016). Ruđer Bošković Institute: Zagreb, 2016;10.

90. Romani $P$, Papi A, Ignesti $M$, et al. Dynamin controls extracellular level of Awd/Nme1 metastasis suppressor protein. Naunyn-Schmiedeberg's Arch Pharmacol 2016;389:1171-1182.

91. Romani $P$, Papi $A$, Ignesti $M$, et al. Awd extracellular levels rely on Dynamin function. In: Edited by Ćetković $\mathrm{H}$, Bosnar $\mathrm{MH}$. 10th International Congress of the NDP Kinase/Nm23/Awd Family (NDPK2016). Ruđer Bošković Institute: Zagreb, 2016; 51.

92. Celniker SE, Dillon LA, Gerstein MB, et al. Unlocking the secrets of the genome. Nature 2009;459:927-930.

93. Lim J, Jang G, Kang S, et al. Cell-permeable NM23 blocks the maintenance and progression of established pulmonary metastasis. Cancer Res 2011;71:7216-7225.

94. Steeg PS, Zollo M, Wieland T. A critical evaluation of biochemical activities reported for the nucleoside diphosphate kinase/Nm23/Awd family proteins: opportunities and missteps in understanding their biological functions. Naunyn-Schmiedeberg's Arch Pharmacol 2011;384:331-339.

95. Hsu T, Steeg PS, Zollo $M$, et al. Progress on Nme (NDP kinase/Nm23/ Awd) gene family-related functions derived from animal model systems: studies on development, cardiovascular disease, and cancer metastasis exemplified. Naunyn-Schmiedeberg's Arch Pharmacol 2015;388:109-117.

96. Brand $\mathrm{AH}$, Perrimon N. Targeted gene expression as a means of altering cell fates and generating dominant phenotypes. Development 1993:118:401-415.

97. Pignoni F, Zipursky SL. Induction of Drosophila eye development by decapentaplegic. Development 1997;124:271-278.

98. Moline MM, Southern C, Bejsovec A. Directionality of wingless protein transport influences epidermal patterning in the Drosophila embryo. Development 1999;126:4375-4384.

99. Lee T, Luo L. Mosaic analysis with a repressible cell marker for studies of gene function in neuronal morphogenesis. Neuron 1999;22: $451-461$. 